\title{
Archéopages
}

Archéopages

Archéologie et société

Hors-série 1 | 2008

Construction ${ }^{\mathrm{s}}$ de l'archéologie

\section{Pour une archéologie du quotidien : objets et céramiques modernes}

\section{Fabienne Ravoire}

\section{(2) OpenEdition}

1 Journals

Édition électronique

URL : https://journals.openedition.org/archeopages/839

DOI : 10.4000/archeopages.839

ISSN : 2269-9872

Éditeur

INRAP - Institut national de recherches archéologiques préventives

Édition imprimée

Date de publication : 1 février 2008

Pagination : $33-35$

ISSN : $1622-8545$

\section{Référence électronique}

Fabienne Ravoire, "Pour une archéologie du quotidien : objets et céramiques modernes »,

Archéopages [En ligne], Hors-série 1 | 2008, mis en ligne le 01 février 2008, consulté le 27 février 2023.

URL : http://journals.openedition.org/archeopages/839; DOI : https://doi.org/10.4000/archeopages.

839

Tous droits réservés 
de moyens d'investigation complémentaires permettant de se rapprocher un peu plus de la réalité de leur œkoumène. Décidément, plonger dans les profondeurs de l'histoire de l'humanité n'est pas une mince affaire...

Remerciements. Nous tenons ici à remercier Hélène Martin et Laurent Bruxelles pour leur concours précieux à la réalisation de cet article.

Bruxelles L., Berthet A.-L., Chalard P., Colonge D., Delfour G., Jarry M., Lelouvier L.-A., Arnoux T., Onézime O. 2003: «Le Paléolithique ancien et moyen en Midi toulousain: nouvelles données et perspectives de l'archéologie préventive», Paléo, ${ }^{\circ} 15$, p. 7-28.

Demoule J.-P. (dir.) 2004: La France archéologique. Vingt ans d'aménagements et de découvertes, Paris, Inrap et Hazan.

DeMOULE J.-P. (dir.) 2007: L'Archéologie préventive dans le monde apports de l'archéologie préventive à la connaissance du passé, Paris, La Découverte.

JARry M., ARramond J.-C. «Identification de sites paléolithiques et mésolithiques en Midi-Pyrénées: méthodes et implications", actes du séminaire Le Diagnostic des sites paléolithiques et mésolithiques, Paris, 5-6 décembre 2006, Les Cahiers de l'Inrap, $\mathrm{n}^{\circ} 3$

Jarry M., Colonge D., Lelouvier L.-A., Mourre V. (dir.) 2007: Les Bosses, Lamagdelaine, Lot, France: un gisement paléolithique moyen antérieur à l'avant-dernier Interglaciaire sur la moyenne terrasse du Lot, Paris, Société préhistorique française (Travaux 7)

JAubert J. 2002: «Le Paléolithique dans le Midi toulousain», in J.-M. Pailler (dir.), Tolosa, nouvelles recherches sur Toulouse et son histoire dans l'Antiquité, Rome, École française de Rome (Coll. de l'École française de Rome 281), p. 43-52.

Jaubert J., Servelle C. 1996: «L'Acheuléen du bassin de la Garonne. Etat de la question et implications », in A. Tuffreau (dir.): "L'Acheuléen dans l'Ouest de l'Europe», actes du colloque international de Saint-Riquier (6-10 juin 1989), Villeneuve-d'Ascq, Centre d'études et de recherches préhistoriques, Université des sciences et technologies de Lille (Publ. du CERP 4), p. 89-106. PICQ P. 2005: Nouvelle Histoire de l'Homme, Paris, Perrin.

VAGINAY M. (dir.), Rousset V. (coll.) 2003: Histoire des sites, histoire des hommes: découvertes archéologiques réalisées lors de la construction de l'autoroute A2o en Quercy, Rodez, Éditions du Rouergue.

À paraître

BRUXelles L., JARRY M., BevilacquA R. «Au-delà des perspectives de l'archéologie préventive pour le renouvellement des données en Midi toulousain: le site paléolithique moyen de Bel-Soleil à Cornebarrieu (Haute-Garonne, France) », in J.-G. Bordes, J. Jaubert, I. Ortega (dir.), « Séance décentralisée de la Société préhistorique française, Bordeaux, 2006: Nouvelles méthodes, nouveaux sites, nouveaux résultats », Bulletin de la Société préhistorique française.

\section{Pour une archéologie du quotidien : objets et céramiques modernes}

\section{Fabienne Ravoire}

Intap, UMR 5594 ARTeHIS

A u-delà de l'objet archéologique.

L'archéologie préventive, en s'ouvrant aux périodes modernes pour lesquelles on dispose d'un grand nombre de sources écrites, offre de nombreuses perspectives de recherche sur l'objet, vecteur privilégié des usages et des pratiques sociales qui ont présidé à son élaboration, à sa fabrication et à sa consommation. Au-delà des études techniques, typologiques et chronologiques qui demeurent d'actualité, il convient désormais d'élargir notre champ d'investigation et de proposer une approche résolument sociale qui tienne compte des données historiques et anthropologiques disponibles. Sous cet angle, il est possible de mettre en place une approche sociale comparative et d'aborder - par-delà les élites pour lesquelles on dispose de sources historiques abondantes et variées - les choix de consommation des populations urbaines et rurales aux revenus modestes. L'objet archéologique permet, en effet, de compenser la faiblesse des sources écrites et apporte un éclairage sociologique fondamental sur la frange démographiquement majoritaire de la population.

Depuis un quart de siècle, les objets trouvés lors de la fouille de sites modernes sont majoritairement en céramique et, dans une moindre mesure, en verre, en métal, en os et en bois. Ils nous renseignent d'abord sur les usages du quotidien, qui vont de la cuisine à la table en passant par la toilette, le jeu, la parure, l'écriture et le commerce (jetons, monnaies), sans oublier le religieux (pot à encens, chapelet, bénitier ). De faible valeur marchande, ces objets, pour la plupart, n'apparaissent pas dans les inventaires enregistrés après décès. L'archéologie montre alors ce qu'ont été les choix notariaux, résultats de tris à la fois économiques et sociologiques variant selon les notaires et les époques. Les hommes - notaires, collectionneurs ou simples usagers - ont établi avec l'objet des relations dépendant de son statut, de ses valeurs marchande et sentimentale; relations qui auront eu tendance à évoluer au gré des conjonctures et des milieux sociologiques. Pour autant, l'objet perdu, jeté, oublié que l'on va retrouver en fouille n'en constituera pas moins un témoin privilégié des usages d'un milieu social à un moment donné de son histoire.

La céramique comme marqueur social. Plus que sur les usages, létude de ces objets doit nous renseigner sur le rôle social qu'ils ont pu jouer, à travers leur mode de consommation, dans la construction identitaire et dans celle des valeurs des élites comme des gens du commun. Prenons l'exemple de la céramique en Île-de-France, pour laquelle on dispose désormais d'un important référentiel spatial, social et chronologique. Nous avons récemment travaillé sur les différenciations sociales que pouvait traduire la céramique du XVI ${ }^{e}$ siècle (Ravoire 2002), puis des XVII ${ }^{e}$ et $\mathrm{XVIII}^{\mathrm{e}}$ siècles (Ravoire 2007b), à partir de plusieurs contextes archéologiques parisiens et franciliens de nature différente.

Les résultats auxquels nous avons abouti confirment le rôle que joue une partie du mobilier céramique dans la compréhension des phénomènes sociaux. Au XVIII siècle, le mobilier des châtelains de Roissy-en-France et des abbesses de Chelles se distingue très nettement de celui des bourgeois de Paris et des fermiers gentilshommes de Varennes. Cependant, au sein des contextes bourgeois et paysans étudiés, les différences observées traduisent moins des écarts de richesses que des décalages culturels. L'étude du mobilier, à travers les sources écrites, aboutit à un résultat similaire: les populations paysannes et urbaines 
se contentent dans leur majorité d'un mobilier limité et fruste (Roche 1997, p. 197). Mais l'objet en céramique peut être également un révélateur de l'évolution des goûts et des modes. Le site de la ferme du Colombier, à Varennes-sur-Seine, en Seine-et-Marne, pour lequel on dispose d'objets archéologiques et d'inventaires après décès, illustre parfaitement ce phénomène de décalage culturel (Ravoire 2006). On a pu constater que les fermiers qui exploitèrent la ferme aux XVII ${ }^{\text {e }}$ et XVIII ${ }^{\text {e }}$ siècles, sans être riches, possédaient un certain nombre de biens en céramique dont la présence dénote une relative aisance matérielle. Aux services de table et aux pots de toilette en faïence retrouvés en fouille s'ajoutaient de la vaisselle d'étain et quelques ustensiles en argent. Cependant, comparés à la bourgeoisie des villes, les fermiers de Varennes ne possédaient aucune des céramiques susceptibles de les classer parmi les gens au fait des dernières modes. Ni le service à thé ou à café ni la porcelaine chinoise - une vaisselle au caractère ostentatoire affirmé - n'ont été retrouvés, ni dans les inventaires après décès ni dans les dépotoirs de la ferme. La vaisselle possédée était essentiellement utilitaire. Le seul luxe de ces paysans résidait en quelques pièces de faïence décorée et d'argenterie tenues serrées en leur armoire.

Les apports de l'analyse spatiale. Dans le droit fil des travaux mis en œuvre en archéologie médiévale par G. Démians d'Archimbaud (Démians d'Archimbaud, Picon 1987), J.-M. Pesez et F. Piponnier (Piponnier 1975), il a également été possible de recourir à l'étude de la répartition spatiale des objets archéologiques pour tenter d'appréhender la fonction des bâtiments mis au jour dans les habitats ruraux. Pour différentes raisons techniques, l'habitat urbain se prête mal à ce type d'étude: les sols de terre battue, susceptibles de piéger les objets, sont en effet absents au profit des sols de plâtre, de pierre et de carreaux; la densité des constructions ne facilite pas la différenciation des unités domestiques ; l'évacuation des déchets, en milieu urbain, ne permet pas de relier ces derniers à l'habitat dont ils sont issus (sauf pour des rejets ponctuels dans des dépotoirs installés à l'arrière de quelques maisons).

En revanche, plusieurs études portant sur des habitats ruraux d'Île-de-France d'époque moderne ont confirmé l'intérêt qu'il y avait à mettre en œuvre ce type de recherche (Ravoire 2007b; Ravoire, à paraître). L'étude de la répartition spatiale du mobilier céramique nous a en effet conduits à porter un regard nouveau sur le fonctionnement de ces sites, qui va bien au-delà des considérations strictement architecturales. La fonction des pièces a pu être appréhendée dès lors que certains récipients, agissant comme de véritables marqueurs sociologiques, ont été retrouvés piégés dans un sol de terre battue ou avaient été rejetés par les ouvertures situées à proximité. C'est ainsi que des récipients culinaires, retrouvés dans une pièce avec foyer, nous ont conduits à reconnaître la cuisine d'une ferme du XVI ${ }^{\mathrm{e}}$ siècle, fouillée à Tremblay (Ravoire, à paraître), tandis que des récipients de conservation et de préparation laitière permettaient d'identifier la laiterie du moulin de Roissy (Ravoire 2007a); cet usage a été confirmé par des sources écrites.

Vers une analyse sociologique. Le mobilier archéologique peut également servir à nuancer l'interprétation que l'on peut faire du degré de confort des habitats ruraux, souvent qualifiés de médiocres par plusieurs générations d'historiens. La présence de chauffe-plats de table - objets qui, au XVI ${ }^{\mathrm{e}}$ siècle, avaient une forte connotation urbaine et bourgeoise - parmi le mobilier exhumé sur ce type d'habitat va à l'encontre de l'image misérabiliste qui nous a été léguée. Un autre exemple significatif des changements que l'étude de la céramique peut apporter à notre perception des intérieurs paysans est celui de la vaisselle en faïence de Nevers, que l'on trouve fréquemment dans les milieux parisiens aisés à partir du XVII ${ }^{\mathrm{e}}$ siècle. Or, cette céramique se retrouve chez le meunier de Roissy, dont on sait par ailleurs qu'il n'avait pas un niveau de vie particulièrement élevé. ${ }^{\mathbf{1}}$ Ce dernier disposait également de beaux verres à pied, identiques à ceux figurés sur l'un des tableaux des frères Le Nain Le Repas de paysans (vers 1642); celui-ci représente une famille de paysans mangeant au coin du feu. Ces découvertes, et bien d'autres de même nature, remettent en question l'idée largement admise, pour ne citer que cet exemple, que «les verres élégants et fragiles sont d'un autre monde» que celui de la paysannerie (Roche 1997, p. 207). Des habitats aussi variés qu'une ferme seigneuriale, un moulin, une ferme du pays de France, une auberge du centre d'Étampes ont en effet révélé des céramiques que l'on s'attendrait, en toute logique, à ne retrouver que dans des demeures aisées. Au regard des distinctions sociologiques affichées par ces différents habitats, il apparaît du point de vue du mobilier archéologique que ce sont moins les productions en tant que telles que la quantité de pièces retrouvées qui les oppose.

L'apport de l'archéologie à une meilleure connaissance des milieux et des clivages sociaux, notamment des paysans d'époque moderne, n'en est qu'à ses débuts. Le développement des fouilles préventives au cours des décennies à venir sera, à n'en pas douter, l'occasion de produire une somme importante de nouvelles connaissances à même de nuancer, voire de modifier radicalement, la vision que les historiens nous ont léguée des sociétés modernes.

Démians d'Archimbaud, G., Picon, M. 1987: Céramiques d'habitat. Réflexion critique sur les données acquises lors des fouilles de Rougiers, in La céramique ( $V^{e}$-XIX ${ }^{e}$ s.), fabrication, commercialisation, utilisation. Actes du colloque de Paris, Caen 1987, p. 245-258.

PIPONNIER, F. 1975 : Une maison paysanne au XIV siècle: le mobilier archéologique, Rotterdam Papers II, p. 151-17o.
1 Information aimablement communiquée par Jean-Yves Dufour, responsable de l'opération archéologique. 
RAVOIRE F. 2002: «Céramique importée et différenciation sociale: l'exemple de la vaisselle parisienne à la Renaissance (fin du Xve $\mathrm{s}$. $\mathrm{XVI}^{\mathrm{e}} \mathrm{s}$.) ", Actes du Colloque international d'Archéologie médiévale et post-médiévale de Bâle (septembre 2002), Bâle, 2002, p. 364-373.

Ravoire F. 2006: «Étude de la céramique de la Ferme du Colombier à Varennes-sur-Seine (XVI ${ }^{\mathrm{e}}-\mathrm{XVIII}^{\mathrm{e}} \mathrm{s}$.) », in S. Hurard, Y. Franzini, Varennes-sur-Seine, "Ferme du Colombier », Rapport final d'opération, Saint-Denis, Service régional de l'archéologie d'île-de-France, Inrap, p. 79-134.

RAVOIRE F. 2007a: «Étude des céramiques retrouvées dans l'habitat et les dépendances du meunier de Roissy-en-France (XVII $-\mathrm{XIX}^{\mathrm{e}} \mathrm{s}$.) », in J.-Y. Dufour, Le site du moulin de Roissy, Rapport final d'opération, Saint-Denis, Service régional de l'archéologie d'Île-de-France, Inrap.

RAVOIRE F. 2007b: «Mobilier céramique des XVII ${ }^{\mathrm{e}}$ et XVIII ${ }^{\mathrm{e}} \mathrm{S}$., approche sociale: l'apport des fouilles archéologiques d'Île-de-France», Actes du Colloque international d'archéologie médiévale et postmédiévale de Paris, Medieval Europe in Paris (Paris, 3-8 septembre 2007); http://medieval-europe-paris-2007.univ-paris1.fr/MEP2007

Roche D. 1997: Histoire des choses banales. Naissance de la consommation $\mathrm{XVII}^{e}$-XIX ${ }^{e}$ siècle, Paris, Fayard.

À paraître

RAVOIRE F. «Évolution du confort matériel dans les maisons paysannes sous l'Ancien Régime en Ile-de-France ( $\mathrm{XVI}^{\mathrm{e}}$-XVIII ${ }^{\mathrm{e}} \mathrm{s}$.) : l'apport des sources archéologiques », in F. Mullane, J.-R. Trochet (dir.), Actes du colloque Les maisons paysannes en Europe occidentale, du la fin du Moyen Âge au XIX ${ }^{e}$ siècle, Université de Paris-Sorbonne, Institut de géographie, Paris, 14-15-16 septembre 2006, Paris, Presses universitaires de Paris-Sorbonne.

\section{Histoire et archéologie des châteaux médiévaux japonais}

Pierre-François Souyri

Université de Genève

L 'émergence d'une archéologie médiévale.

Depuis une trentaine d'années,

la connaissance de la société médiévale japonaise a considérablement évolué, avec de nouveaux questionnements liés à la pratique grandissante de l'interdisciplinarité.

Les rapprochements intellectuels, notamment entre historiens des textes et archéologues, se sont révélés particulièrement féconds. Jusque dans les années 1960-1970, l'archéologie (discipline qui $\mathrm{a}$, dans l'archipel, plus d'un siècle d'histoire) ne s'intéressait qu'aux sociétés préhistoriques et protohistoriques. Depuis, elle a élargi son champ de recherches aux périodes historiques anciennes et médiévales, notamment sous l'impact des lois qui, depuis les années 1970, structurent l'étude que l'on désigne au Japon par les termes de «patrimoine enfoui». Cette législation a permis la multiplication des fouilles d'urgence et une sensibilisation plus grande des collectivités locales aux retombées économiques éventuelles de «découvertes » archéologiques majeures.

La grande tradition pluriséculaire japonaise d'interrogation des sources anciennes écrites a été bouleversée par l'exhumation, à partir des années 1960, de mokkan - tablettes en bois sur lesquelles ont été rédigées à l'encre des indications relatives au fonctionnement de la société. Les plus anciennes de ces tablettes remontent aux $\mathrm{VII}^{\mathrm{e}}$ et $\mathrm{VIII}^{\mathrm{e}}$ siècles et ont surtout été découvertes dans les anciens palais et capitales du début de la période historique ancienne. Certaines ont également été retrouvées dans des sites plus récents et plus modestes, notamment des agglomérations et bourgades situées le long des routes (comme Mitsuke, daté des $\mathrm{XIV}^{\mathrm{e}}$ et $\mathrm{XV}^{\mathrm{e}}$ siècles) ou à l'embouchure de rivières (comme Kusado Sengen, daté des $\mathrm{XV}^{\mathrm{e}}$ et $\mathrm{XVI}^{\mathrm{e}}$ siècles). Sur ces tablettes, on peut déchiffrer des ex-voto, des listes de marchandises en vente, des prix, etc. En mettant au jour ces documents d'un type inédit, les archéologues ont acquis un nouveau statut aux yeux des historiens, celui de «découvreurs» de sources écrites d'un nouveau genre. Ces tablettes permettent en effet de faire renaître des pans entiers de l'activité humaine d'autrefois que les sources écrites traditionnelles ignoraient jusqu'alors, en particulier dans le domaine de l'histoire économique: celle des échanges et du commerce.

Associée à la multiplication des chantiers liée aux fouilles de sauvetage et à la mise en place de grands chantiers programmés, l'archéologie médiévale est en conséquence devenue productrice de sources nouvelles et plus seulement de sources écrites. Dans deux secteurs au moins, les recherches archéologiques ont permis de modifier l'idée que l'on se faisait de la société médiévale, tant pour ce qui concerne l'histoire urbaine (tracés des voies de communication, habitat, centres religieux, activités économiques, étude anthropologique de l'espace urbain, etc.) que l'histoire des châteaux.

Des mottes castrales aux châteaux de pierre. Tout le monde connaît de ces châteaux japonais aux formes imposantes et, à nos yeux, exotiques. En fait, ces constructions sont récentes et remontent à la fin du XVI ${ }^{\mathrm{e}}$ siècle, voire, le plus souvent, au début du XVII ${ }^{\mathrm{e}}$ siècle. Toujours en place et bien documentées, ces constructions castrales ont évidemment suscité l'intérêt des historiens et des architectes. Mais ce qui est moins connu, c'est l'existence bien avant le XVII ${ }^{\mathrm{e}}$ siècle de châteaux ou de résidences castrales liés à la naissance et au développement d'une classe guerrière: les fameux samouraïs. Ces constructions, dont les superstructures étaient en bois, n'ont pas résisté au temps ; il n'en existe guère de traces visibles, au contraire des châteaux forts européens dont les ruines surplombent parfois les vallées. Au Japon, les choses sont bien moins spectaculaires : il ne subsiste, le plus souvent, que des traces de remblais, de fossés ou d'enceintes, fréquemment recouvertes d'une végétation inextricable [Fig.1].

Du nord du Hokkaidô jusqu'aux îles tropicales d'Okinawa, on a repéré près de quarante mille sites castraux anciens, avec, dans certaines vallées, un site attesté presque tous les kilomètres. Parfois, ces sites ressemblent à s'y méprendre aux mottes castrales européennes, si ce n'est le couvert végétal serré de bambous et de sylve à feuilles persistantes venant nous rappeler que nous sommes dans l'archipel... Cette densité est à la mesure de l'intensité du phénomène de la guerre dans le Japon médiéval. Elle souligne aussi l'importance historique d'une classe de guerriers dont on pense qu'elle a pu représenter 